\section{Boxing clever: \\ unravelling Boxer dog health in the UK using primary-care veterinary records}

\section{Kate Barrett, Dave C. Brodbelt, David B. Church, Dan G. O'Neill}

Royal Veterinary College, Hertfordshire, United Kingdom

\section{OBJECTIVES}

The Boxer dog is reportedly predisposed to many disorders but these reports (often decades-old or non-UK) generalize to the current UK Boxer dog population.

\section{METHODS}

Demographic, disorder and mortality data were extracted from VetCompass ${ }^{\top M}$ anonymized clinical records on Boxer dogs under UK primary veterinary care during 2016. We hypothesized the prevalence of ulcerative keratitis is higher in Boxer dogs aged $\geq 6$ years than in those aged $<6$ years'.

\section{RESULTS}

This study comprised 3219 Boxer dogs (0.96\% of all dogs). Median age was 5.81 years (IQR 3.10-8.79, range 0.18 18.37). Median adult bodyweight was $29.9 \mathrm{~kg}$ (IQR 26.333.9, range 15.1-52.5); males were heavier $(32.4 \mathrm{~kg}, \mathrm{IQR}$ $29.3-36.5)$ than females (27.0 kg, IQR 24.4-30.3) ( $<<0.001)$.

The most common disorders were otitis externa (7.14\%, 95\% Cl: $6.31-8.09)$, epulis $(5.84 \%, 95 \% \mathrm{Cl}: 5.08-$ $6.70)$ and ulcerative keratitis $(5.00 \%, 95 \% \mathrm{Cl}: 4.30-5.81)$.

Boxer dogs aged $\geq 6$ years had 13.6 times the odds of ulcerative keratitis ( $95 \% \mathrm{Cl}: 7.5-24.7$ and $\mathrm{p}<0.001) \mathrm{com}-$ pared with those aged $<6$ years.

Median longevity of 346 Boxer dog deaths was 10.57 years (IQR 8.90-12.19, range 1.02-18.37). The most common grouped causes of death were neoplasia $(15.8 \%, 95 \%$ $\mathrm{Cl}: 11.91-20.54)$, brain disorder $(12.1 \%$, $95 \% \mathrm{Cl}: 8.74-$ 16.49) and mass lesions (10.6\%, 95\% Cl: $7.50-14.84)$.

\section{STATEMENT (CONCLUSIONS)}

We identified and distinguished common disorders, e.g otitis externa, from predisposed disorders, e.g. epulis and ulcerative keratitis (increasing predisposition over 6 years old) in the current UK general Boxer dog population. This perspective can assist veterinarians to prioritise their treatments and advisory activities.
Clinical usage of gabapentin in dogs under primary veterinary care in the UK

\section{Lavinia F Mitton, Sandra Sanchis-Mora, Ludovic Pelligand, Holger Volk, David C Brodbelt, Dan G O'Neill}

Royal Veterinary College, Hertfordshire, United Kingdom

\section{OBJECTIVES}

This retrospective study aimed to explore gabapentin use in dogs in the UK. We aimed to report usage prevalence, conditions treated, therapeutic effectiveness, treatment duration and dose regimens, suspected adverse reactions and accompanying treatments.

\section{METHODS}

The study included 318,922 dogs within the VetCompass ${ }^{\mathrm{TM}}$ Program under primary veterinary care in 2016. Data were collected from anonymised electronic medical records.

\section{RESULTS}

Usage prevalence of gabapentin was $0.44 \%(95 \% \mathrm{Cl}$ : $0.42-0.47 \% ; n=1415)$. Twenty condition types were treated including: spinal pain and/or lesion (31.7\%), pain musculoskeletal (23.9\%), osteoarthritis (13.2\%), Chiarilike malformation/syringomyelia (4.7\%), intervertebral disc disease (4.3\%), unspecified neuromuscular signs (4.0\%), unspecified pain $(3.8 \%)$, cruciate disease $(2.7 \%)$ and seizure(s) (1.9\%). Gabapentin was reported to improve clinical signs in $47.3 \%$ of dogs. The course duration was generally short term $(59.7 \%$; $<30$ days between first and last dispense) and $59.6 \%$ of doses were given twice daily. The prevalence of suspected adverse reactions was $7.85 \%(95 \% \mathrm{Cl}: 6.57 \%-9.36 \% ; n=1438)$ and the most common were sedation $(5.2 \%)$ and ataxia (1.3\%). The most common accompanying treatments alongside gabapentin were non-steroidal anti-inflammatory drugs (27.2\%), tramadol (9.0\%), glucocorticoids (5.6\%) and opioids $(4.8 \%)$.

\section{STATEMENT (CONCLUSIONS)}

This study provides useful benchmarking data on gabapentin usage in primary-care practice and suggests a low occurrence of mild side effects. Despite the subjective nature of the efficacy assessment, gabapentin showed a good therapeutic efficacy. These results can assist practicing clinicians to benchmark their clinical use of gabapentin and to predict suspected adverse reactions. 